From Proc. IFAC CACSD'97, Gent, Belgium, 28-30 April 1997. CIFAC

\title{
ENHANCED MATLAB TOOLS FOR LINEAR AND NONLINEAR SYSTEM STABILILTY
}

\author{
James H. Taylor \& Cheney Chan
}

\author{
Department of Electrical Engineering \\ University of New Brunswick \\ PO Box 4400, Fredericton, NB CANADA E3B $5 A 3$ \\ email: jtaylor@unb.ca
}

\begin{abstract}
Two Matlab-based tools have been developed for the convenient assessment of stability conditions for linear and nonlinear systems. The first is a more helpful and definitive version of nyquist, which has features of automatic zooming (to show all crossings of the real axis and thus display the corresponding critical gain points) and which displays on the real axis a numeral (' 0 ', ' 1 ' etc.) that represents the number of unstable (right-half plane) poles that will result in a closed-loop system with feedback gain $k$ if $-1 / k$ is located in that region. The second tool is built on this extended nyquist command, and makes the application of the nonlinear time-varying system Circle Criterion equally simple and definitive. Examples are presented to show the efficacy of these extensions within the MATLAB environment.
\end{abstract}

Keywords: Stability; linear systems; nonlinear systems; Nyquist Criterion; Circle Criterion.

\section{Introduction}

Existing MATLAB commands for the stability analysis of linear systems are not easy to interpret by the less-than-expert user, and tools for assessing the stability of nonlinear systems are nonexistent in MATLAB. In the linear case, interpreting the usual MATLAB Nyquist plot is not hard if the plant is stable and the $W(j \omega)$ locus is not complicated, but it may be confusing, especially if there are multiple real-axis crossings and/or openloop poles on the imaginary axis or in the righthalf plane (RHP). In the nonlinear case, none of the classical absolute stability criteria are implemented in MATLAB, and we have addressed that lack by creating a comparably user-friendly tool for the application of the Circle Criterion $[1,2]$.

\section{Stability Criteria}

In this section we state the linear and nonlinear stability criteria, emphasizing their classical geometric interpretations. As a preliminary, the class of systems considered is depicted in Fig. 1, where it is assumed that the forward-path transfer function is expressed in state-space form as:

$$
W(s)=C(s I-A)^{-1} B+D
$$

or in Laplace notation as a ratio of polynomials, with the order of the numerator not exceeding that of the denominator:

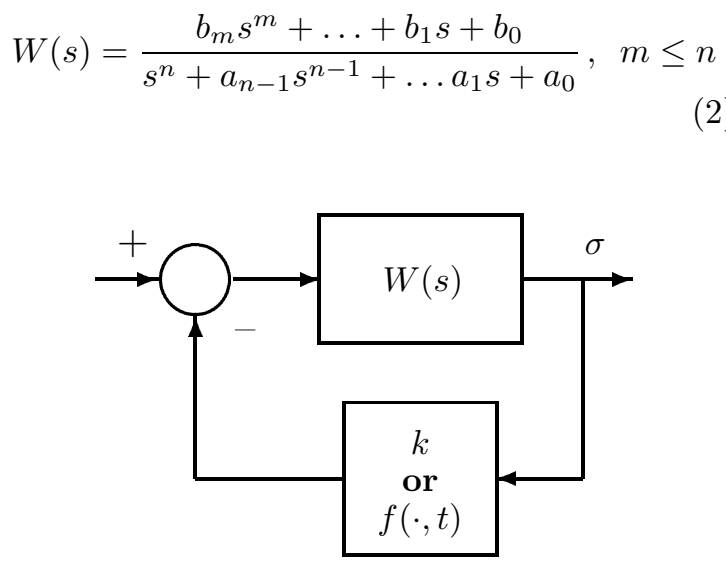

Figure 1: Closed-loop Linear/Nonlinear System

The Nyquist criterion states that the closed-loop system in Fig. 1 with $k$ in the feedback path will be stable if the point $-1 / k$ is not in the $W(s)$-map of the right-half of the $s$-plane (RHP). In the case of a stable plant $W(s)$, this map is simply that region to the right as one traverses the Nyquist plot of $W(j \omega)$. In the case of a plant with $q$ poles in the right-half plane, this map must take into consideration $q$ map layers due to excluding these singularities by traversing a small circle around each in the counter-clockwise sense, which results in a large circle traversed in the clockwise sense for each. As an example, consider the unstable plant:

$$
W(s)=\frac{s+2}{s^{2}-4 s-5}
$$

Then the $s$-plane region being mapped is depicted in Fig. 2 and the resulting complete Nyquist diagram is portrayed in Fig. 3. Observe that the only range of $k$ leading to stability is $4<k<\infty$; the corresponding region for $-1 / k$ is shown with the 


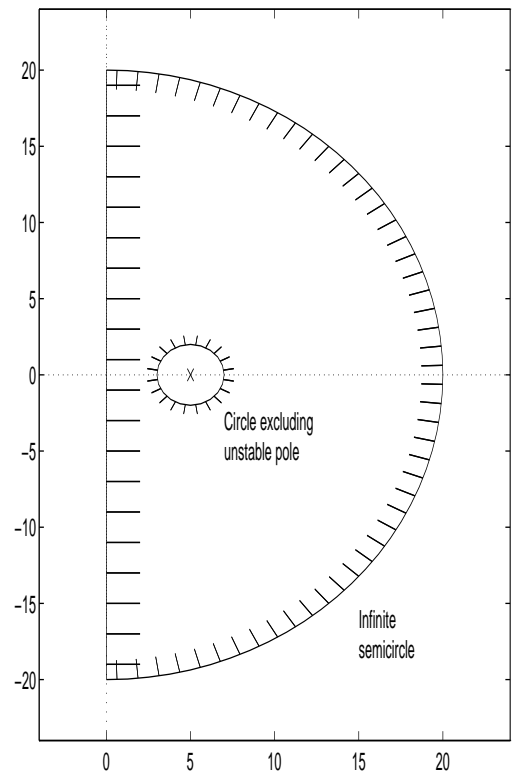

Figure 2: $s$-plane region mapped for Nyquist criterion

numeral 0 on the real axis to denote no closedloop poles in the RHP. For $2.5<k<4$ there will be 2 unstable closed-loop poles, and for the range $-\infty<k<2.5$ there will be one; again, the numerals on the real axis indicate these results.

The Circle Criterion [1, 2] states that the closedloop system in Fig. 1 with $f(\cdot, t)$ in the sector $[\underline{F}, \bar{F}]$, i.e.,

$$
\underline{F} \leq \frac{f(\sigma, t)}{\sigma} \leq \bar{F}
$$

is absolutely stable (uniformly asymptotically stable in the large - UASIL) if one can draw a circle on the $W=U+j V$ plane whose diameter is defined by the points $V=0,-1 / \underline{F}<U<-1 / \bar{F}$ and whose interior has no points in common with the $W(s)$-map of the right-half of the $s$-plane. In essence, this circle must lie in a region of the Nyquist plane where there are zero mapping layers in the sense of the Nyquist criterion stated above. The case $\underline{F}<0<\bar{F}$ is interesting in that the "diameter" so defined passes through the point $\infty$ and the "inside" of the circle is actually what would ordinarily be considered the outside (exterior points), as shall be illustrated in the examples of Section 4.

\section{Description of MATLAB Stability Routines}

Given the above problem definition, the following "help" displays (reformatted to fit the columns of the IFAC style) constitute a concise "users' manual" for the routines newnyq and circle:

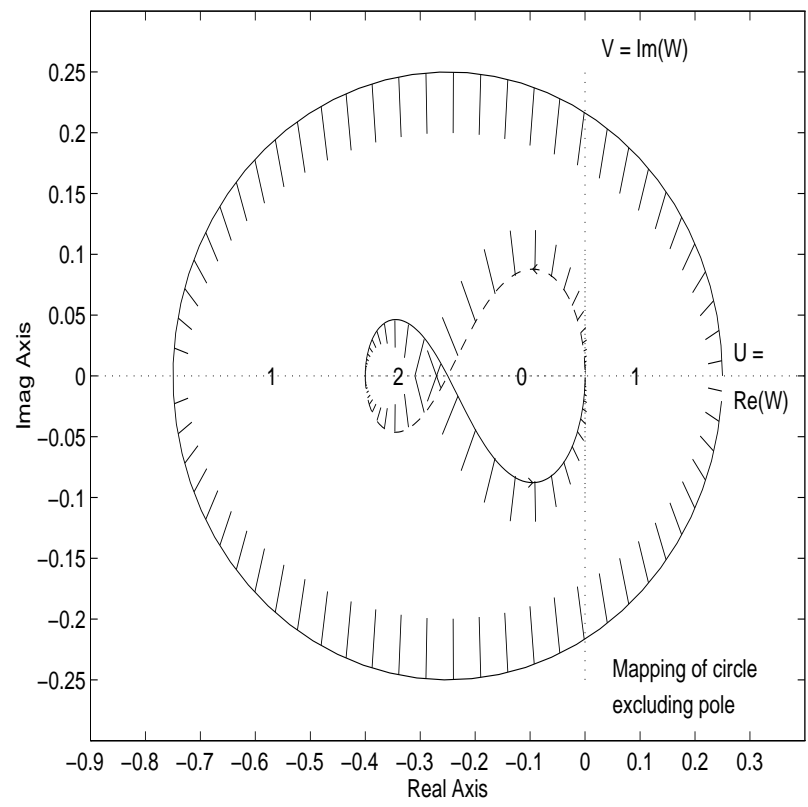

Figure 3: $W(s)$-map for the Nyquist criterion

\section{NEWNYQ}

A new and improved version of Nyquist frequency response for SISO continuous-time linear systems (see NYQUIST). NEWNYQ(A,B,C,D) produces a Nyquist plot. The frequency range, number of points and scaling are set automatically, and a "zoom" feature displays all real-axis crossings. In addition, the $k$ range(s) for closed-loop stability are reported, and the number of unstable closedloop poles for $-1 / k$ on various regions of the real axis are displayed.

NEWNYQ(A,B,C,D,S) will cause the right-half plane mapping of $W(s)=C(s I-A)^{-1} B+D$ to be indicated by hatching.

NEWNYQ(NUM,DEN) and NEWNYQ(NUM,DEN,S) are corresponding variants for $W(s)$ provided in ratio of polynomial form.

CIRCLE

An implementation of the Circle Criterion for nonlinear feedback control systems with a linear part $W(s)$ and a nonlinearity lying in a sector $\left[F_{\min }, F_{\max }\right]$, i.e.,

$$
F_{\min } \leq f(\sigma, t) / \sigma \leq F_{\max }
$$

The nonlinear system is uniformly asymptotically stable in the large (UASIL) if the circle whose diameter is defined by the points $-1 / F_{\max } \leq$ $\Re(W(j \omega)) \leq-1 / F_{\text {min }}$ has no interior points in the RHP mapping of $W(s)$.

CIRCLE(A,B,C,D,F_MIN,F_MAX) will check if the $\mathrm{CC}$ is satisfied for the sector-bounded nonlinearity and $W(s)=C(s I-A)^{-1} B+D$ 
CIRCLE(A,B,C,D,F_MIN) will determine the

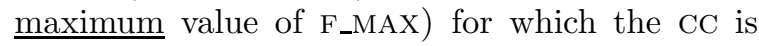
satisfied.

CIRCLE(A,B,C,D,NaN,F_MAX) will determine the

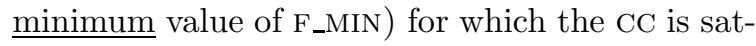
isfied.

CIRCLE(NUM,DEN,F_MIN,F_MAX) etc. are corresponding forms for $W(s)$ provided in ratio of polynomial form.

This routine is built on NEWNYQ, a new and improved version of the Nyquist criterion with advanced graphics and reporting.

\section{Examples}

First we illustrate the use of newnyq and circle on a relatively simple stable plant:

$$
W(s)=\frac{s+1}{s^{4}+2 s^{3}+25 s^{2}+3 s+1}
$$
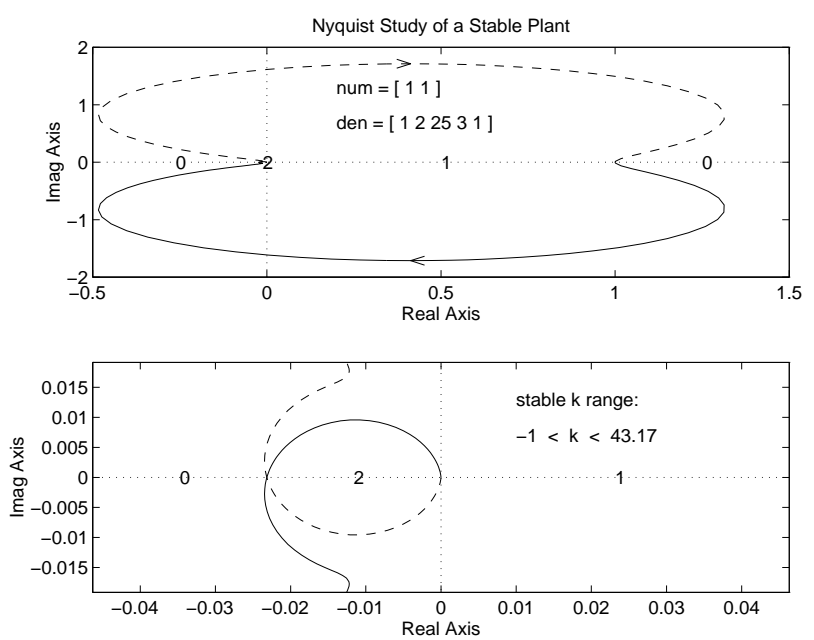

Figure 4: Nyquist Criterion Example (Stable Plant, with Zooming)

Note that the upper plot is similar to that produced by the MATLAB nyquist command except for the numerals on the real axis indicating the corresponding number of unstable closed-loop poles. The lower plot in Fig. 4 illustrates the feature of automatic zooming (to show all crossings of the real axis); the only manual operations in producing this figure were the text commands to document the problem (record num and den and the stable $k$ range). The report that newnyq provides after it is invoked for this problem is:

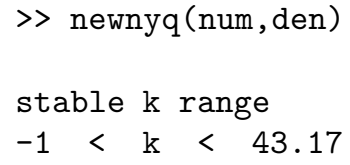

Finally, the numerals $0,1,2$ written on the real axis represent the number of right-half-plane closed-loop poles that would result if $-1 / k$ were to lie in each region.

Once the Nyquist plot has been viewed, one may request that the CC be applied to the same $W(j \omega)$ and a lower sector bound of $F_{\min }=2.5$. The CC

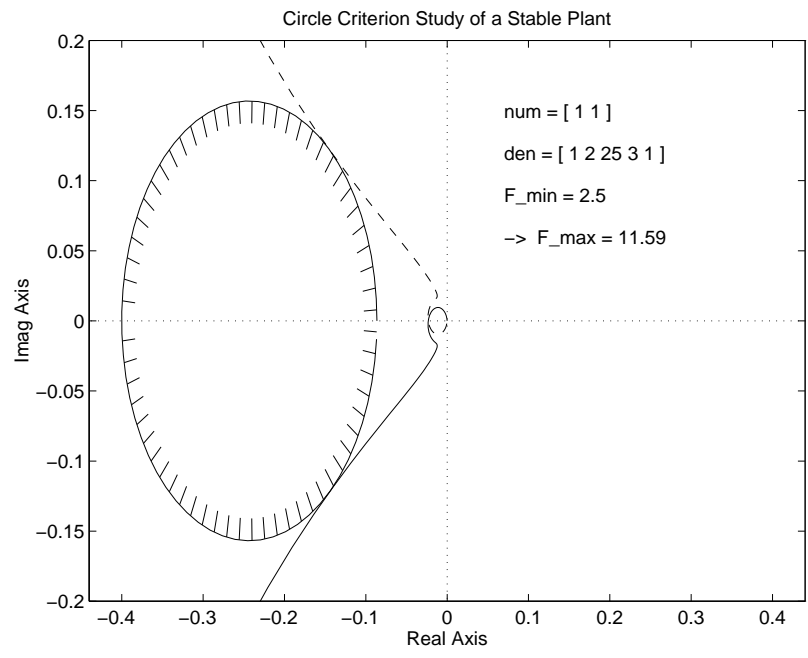

Figure 5: Circle Criterion Example (Stable Plant)

locus is shown on the Nyquist plot (Fig. 5), and the report that circle provides after it is invoked for this problem is:

> circle (num, den, 2.5)

stable $\mathrm{k}$ range

$-1<\mathrm{k}<43.17$

circle criterion is satisfied

maximum sector bound $F_{-} \max =11.59$

(note that it includes the newnyq report, as shown above). Again - the only manual steps in preparing Fig. 5 were the documentation num $=$, den $=, F$ min $=$ etc. defining the problem and the result. The interpretation of this result is that $f(\cdot, t)$ in Fig. 1 must lie in the sector [2.5, 11.59 ], i.e.,

$$
2.5 \leq \frac{f(\sigma, t)}{\sigma} \leq 11.69
$$

in order to guarantee absolute stability.

We conclude this example by illustrating the observation in Section 2 that the "interior" of the CC circle may include the point $\infty$ by taking $F_{\text {min }}=-0.5$ for the above plant. The result is shown in the following report and Fig. 6:

>> circle (num, den, -0.5)

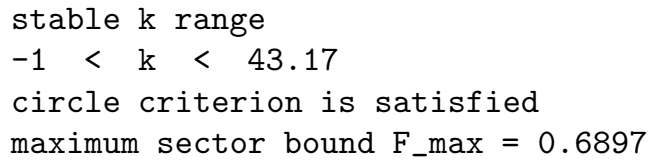




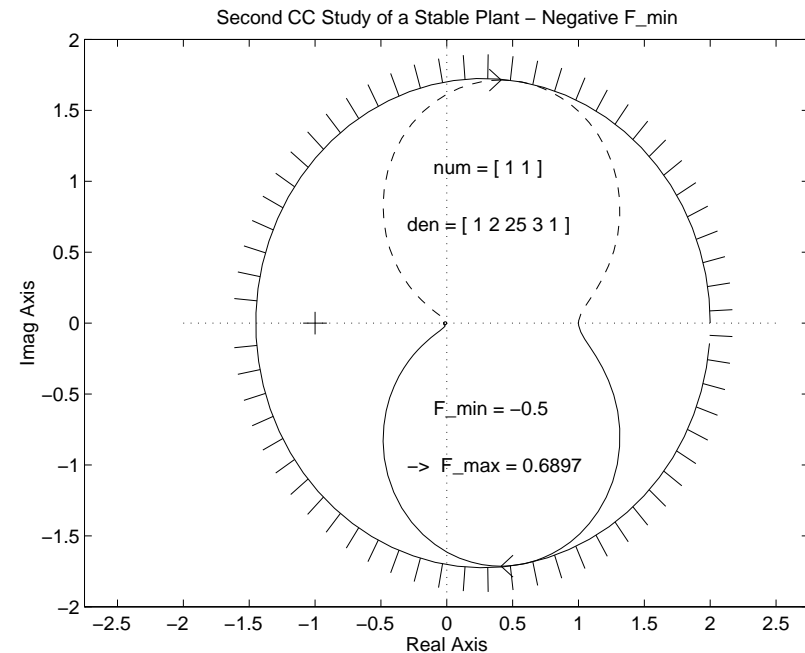

Figure 6: Circle Criterion Result for Negative F_min

Next, we provide the MATLAB plots obtained applying newnyq and circle to the unstable plant in Eqn. 3. The resulting "new Nyquist" plot is

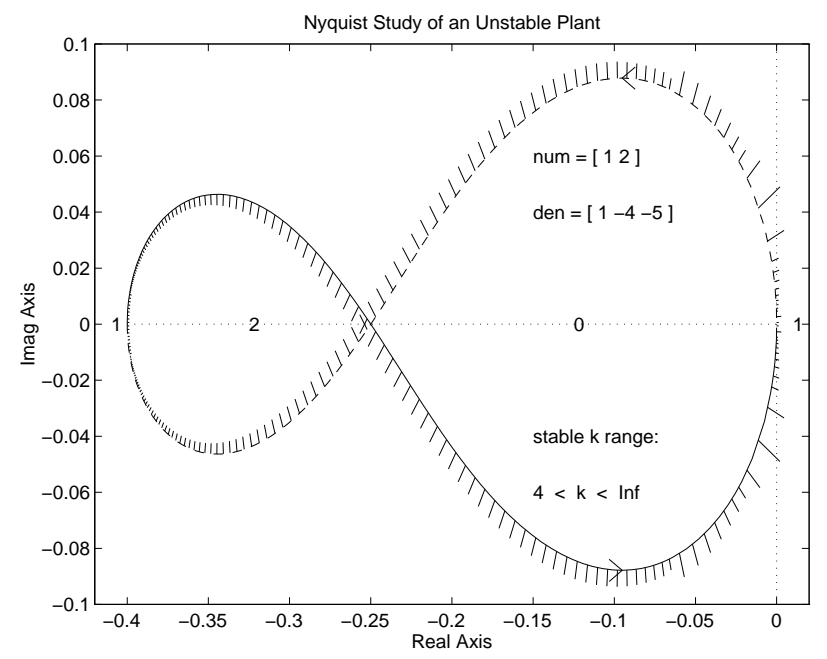

Figure 7: Nyquist Criterion Example (Unstable Plant)

depicted in Fig. 7; note that it is similar to Fig. 3 except that the "large (infinite) circle" arising from the exclusion of the right-half-plane singularity is not shown (to avoid unnecessary clutter); that circle is, however, the basis for the numeral 1 at the far left on the real axis (meaning that any $k$ such that $-1 / k<-0.4$ will result in a closed-loop system with one unstable pole). The region $-0.25<-1 / k<0$ on the real axis has the numeral 0 , because the points are to the left of the $W(j \omega)$ locus meaning that points there are no longer in the right-half-plane map of $W(s)$. In light of this, the report that newnyq provides in this case is: stable $\mathrm{k}$ range

$4<\mathrm{k}<\operatorname{Inf}$

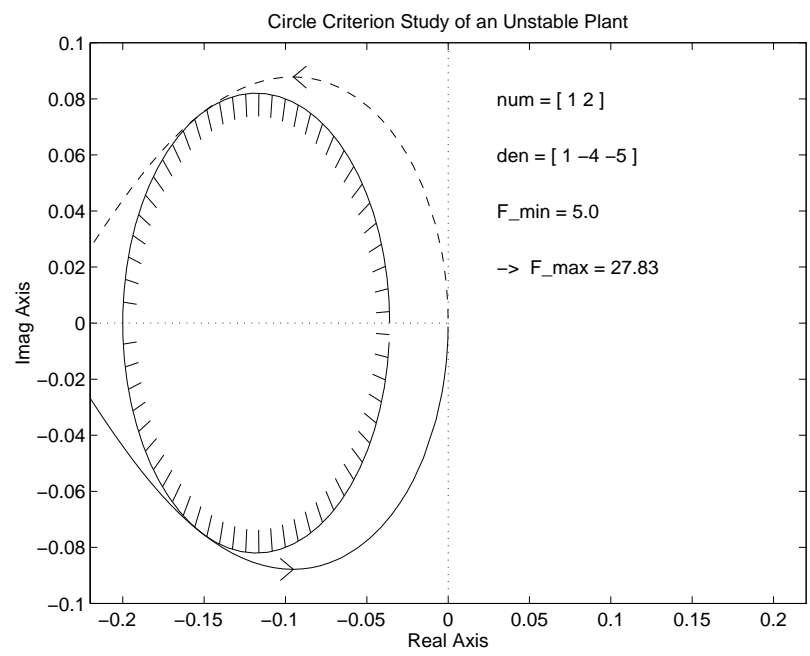

Figure 8: Circle Criterion Example (Unstable Plant)

To illustrate the application of the Circle Criterion tool to the same unstable plant, we specify a lower bound of $\underline{F}=5$, which is in the Nyquist range as required, and the report that circle provides for this problem is:

>> circle (num, den, 5)

stable $\mathrm{k}$ range

$4<\mathrm{k}<\operatorname{Inf}$

circle criterion is satisfied

maximum sector bound $F_{-} \max =27.83$

Again, we note that the corresponding circle in the Nyquist plane lies completely within the "no mapping" portion of the plot.

Another feature of circle allows us to specify the upper bound $\left(F_{\max }\right)$ and obtain the minimum corresponding lower bound that guarantees UASIL. To demonstrate this we issue the command circle (num, den, $\mathrm{NaN}, 100$ ) on the above unstable plant. We receive the following report and plot:

>> circle (num, den, NaN, 100)

stable $\mathrm{k}$ range

$4<\mathrm{k}<\operatorname{Inf}$

circle criterion is satisfied

minimum sector bound F_min $=5.392$ 


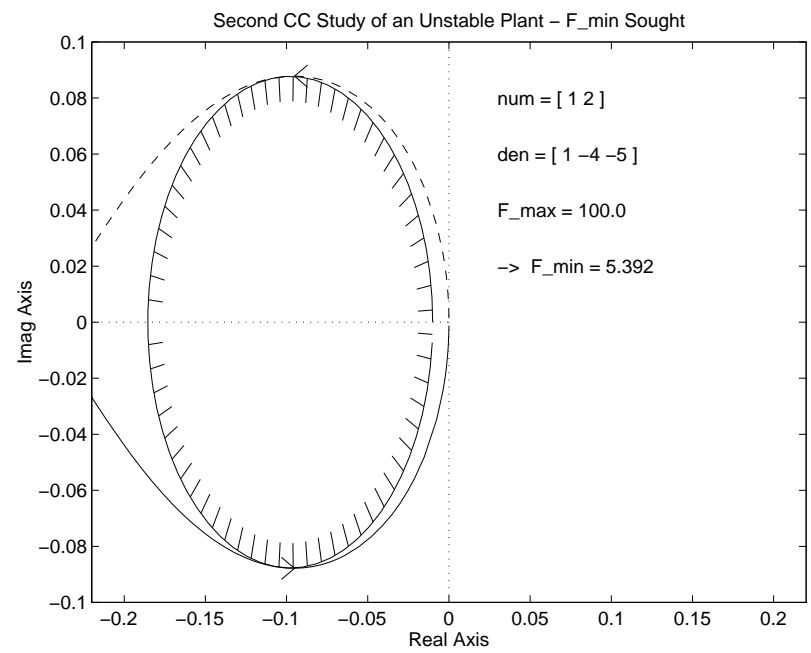

Figure 9: Circle Criterion Example (Upper Bound Provided)

\section{Conclusion}

The routines presented above provide a simple environment for the determination of stability conditions for linear and nonlinear plants. We trust that the convenience and added support they supply can be readily appreciated from the examples presented in Section 4. We have installed these routines on our web page, URL $=$ http://www.ee.unb.ca/jtaylor/ for your access. A copy of this paper is also available there, to serve as a small "User's Guide".

\section{References}

[1] Narendra, K. S. and Goldwyn, R. M. "A Geometrical Criterion for the Stability of Certain Nonlinear Nonautonomous Systems", IEEE Trans. Circuit Theory, CT-11, No. 3, 1964.

[2] Narendra, K. S. and Taylor, J. H. Frequency Domain Criteria for Absolute Stability, Academic Press Inc., Reading, MA, 1973. 